\title{
Optimal Distribution-free Confidence Bands for a Distribution Function
}

\author{
Jesse Frey *
}

\begin{abstract}
Distribution-free confidence bands for a distribution function are typically obtained by inverting a distribution-free hypothesis test. We propose an alternate strategy in which the upper and lower bounds of the confidence band are chosen to minimize a narrowness criterion. We derive necessary and sufficient conditions for optimality with respect to such a criterion, and we use these conditions to construct an algorithm for finding optimal bands. We also derive uniqueness results, with the Brunn-Minkowski Inequality from the theory of convex bodies playing a key role in this work. We illustrate the optimal confidence bands using some galaxy velocity data, and we also show that the optimal bands compare favorably to other bands both in terms of power and in terms of area enclosed.

KEY WORDS: Brunn-Minkowski Inequality; Kolmogorov-Smirnov; Noé's recursion; Nonparametric likelihood
\end{abstract}

\footnotetext{
*Jesse Frey is Assistant Professor, Department of Mathematical Sciences, Villanova University, Villanova, PA 19085. E-mail: jesse.frey@villanova.edu
} 


\section{Introduction}

Given an iid sample $X_{1}, \ldots, X_{n}$ from an unknown continuous distribution $F$, the natural nonparametric estimate of $F$ is the empirical distribution function (EDF), given by

$$
F_{n}(x)=\frac{1}{n} \sum_{i=1}^{n} I\left(X_{i} \leq x\right) .
$$

Since $F_{n}$ is only a point estimate, however, we often supplement it by providing a distribution-free confidence band for the unknown distribution function. These distribution-free confidence bands are typically obtained by inverting a distributionfree hypothesis test of $H_{0}: F=F_{0}$ versus $H_{1}: F \neq F_{0}$. The best-known bands are based on the Kolmogorov-Smirnov statistic

$$
D=\sup _{x}\left|F_{0}(x)-F_{n}(x)\right|
$$

and they have lower and upper bounds of the form

$$
L(x)=\max \left\{0, F_{n}(x)-c\right\} \text { and } U(x)=\min \left\{1, F_{n}(x)+c\right\}
$$

where $c$ is the critical value for the test.

Since confidence bands of the Kolmogorov-Smirnov type are generally perceived as undesirably wide in the tails of the distribution, a number of competitors have been proposed in the literature. Weighted versions

$$
D_{\psi}=\sup _{x} \psi\left(F_{0}(x)\right)\left|F_{0}(x)-F_{n}(x)\right|
$$

of the Kolmogorov-Smirnov statistic were studied by Anderson and Darling (1952), and inverting the test based on $D_{\psi}$ with $\psi(x)=(x(1-x))^{-1 / 2}$ yields what is now 
known as the Anderson-Darling confidence band. More recently, Owen (1995) proposed the use of nonparametric likelihood confidence bands obtained by inverting a distribution-free test studied by Berk and Jones (1979). In this case, the test statistic is given by

$$
L=\inf _{x} K\left(F_{0}(x), F_{n}(x)\right)
$$

where $K\left(F_{0}(x), F_{n}(x)\right) \leq 1$ is the pointwise likelihood ratio statistic for testing $H_{0}$ : $F(x)=F_{0}(x)$ against $H_{1}: F(x) \neq F_{0}(x)$.

Each of these distribution-free confidence bands has upper and lower bounds that are step functions, with the jumps occurring at observed data values. They also have a number of nice properties. For example, the Anderson-Darling confidence band is known to have good power against heavy-tailed alternatives, and the nonparametric likelihood confidence band is known to have, in a certain sense, asymptotically better power than any band based on a weighted Kolmogorov-Smirnov statistic $D_{\psi}$ (see Owen 1995). What these bands lack, however, is small-sample optimality in any nontrivial sense. The emphasis on power that motivates the creation of these bands may also make them less than ideal for use as exploratory analysis tools.

In this paper, we depart from the usual strategy of obtaining confidence bands via test inversion. Instead, we propose an alternate strategy in which the upper and lower bounds of the confidence band are chosen to minimize one of a family of narrowness criteria. Optimization is carried out within a large class of distributionfree confidence bands that includes all the confidence bands we have described. The optimality criteria we use are based on the differences between the upper and lower 
bounds of the band on each interval between observed data values. These criteria may be interpreted in terms of expected area, and they may also be tailored so that special emphasis is put on obtaining bands that are narrow in particular regions (e.g., the tails or the center) of the distribution.

In Section 2, we describe the large class of distribution-free confidence bands over which the optimization is done. We also explain how coverage probabilities are computed. In Section 3, we define a family of optimality criteria, and in Section 4, we derive the theoretical results needed for recognizing optimal bands within that family. In Section 5, we describe an algorithm for computing optimal bands. In Section 6, we compare the optimal bands to other bands in the literature both in terms of power and in terms of shape. One comparison uses a galaxy velocity dataset. In Section 7, we state our conclusions.

\section{A Class of Distribution-free Confidence Bands}

The class of distribution-free confidence bands that we consider is the set of all bands with lower and upper bounds that are step functions with jumps only at observed data values. We set notation as follows. Let $X_{1}, \ldots, X_{n}$ be an iid sample from the distribution with distribution function $F$. Let $X_{(1)}, \ldots, X_{(n)}$ be the order statistics corresponding to $X_{1}, \ldots, X_{n}$, and set $X_{(0)} \equiv-\infty$ and $X_{(n+1)} \equiv \infty$. Let the vectors $a=\left(a_{0}, a_{1}, \ldots, a_{n}\right)$ and $b=\left(b_{0}, b_{1}, \ldots, b_{n}\right)$ be vectors of values from the interval $[0,1]$ and let $\mathcal{F}$ be the set of all distribution functions. We can then define a confidence 
band $C B(a, b)$ by setting

$$
C B(a, b) \equiv\left\{G \in \mathcal{F}: a_{i} \leq G(x) \leq b_{i} \forall x \in\left[X_{(i)}, X_{(i+1)}\right), i=0, \ldots, n\right\}
$$

Since $\lim _{x \rightarrow \infty} F(x)=1$ and $\lim _{x \rightarrow-\infty} F(x)=0$ for all $F \in \mathcal{F}$, the confidence band will have coverage probability 0 if $a_{0}>0$ or $b_{n}<1$. Moreover, if $a_{i+1}<a_{i}$ for some $i$, then the coverage probability of the band will be unchanged if we increase $a_{i+1}$ to $a_{i}$, and if $b_{i+1}<b_{i}$ for some $i$, then the coverage probability will be unchanged if we decrease $b_{i}$ to $b_{i+1}$. Since it is obviously desirable to make the band narrower when there is no corresponding decrease in the coverage probability, we may take the bounds $a_{0}, \ldots, a_{n}$ and $b_{0}, \ldots, b_{n}$ to be ordered as $0 \leq a_{0} \leq \cdots \leq a_{n} \leq 1$ and $0 \leq b_{0} \leq \cdots \leq b_{n} \leq 1$. Finally, if $F$ is continuous, the coverage probability of the band will necessarily be 0 unless $a_{i} \leq b_{i-1}$ for $i=1, \ldots, n$ so that the intervals $\left(a_{i-1}, b_{i-1}\right)$ and $\left(a_{i}, b_{i}\right)$ always overlap. It thus suffices for optimization purposes to consider only confidence bands $C B(a, b)$ for which the vectors of bounds $a$ and $b$ satisfy the conditions

$$
\begin{gathered}
0=a_{0} \leq a_{1} \leq \ldots a_{n} \leq 1,0 \leq b_{0} \leq \ldots \leq b_{n}=1, \quad \text { and } \\
a_{i} \leq b_{i-1}, i=1, \ldots, n
\end{gathered}
$$

Taking $a_{0}=0$ and $b_{n}=1$ as given, we define a collection of vectors of bounds as

$$
V=\left\{(a, b)=\left(a_{1}, \ldots, a_{n}, b_{0}, \ldots, b_{n-1}\right):(a, b) \in[0,1]^{2 n} \text { and }(a, b) \text { satisfies }(1)\right\}
$$

The class of confidence bands over which we will optimize is then given by

$$
\mathcal{B}_{V}=\{C B(a, b):(a, b) \in V\}
$$


One should note that the randomness of the band $C B(a, b)$ arises from the randomness in the values $X_{(1)}, \ldots, X_{(n)}$ and not from any uncertainty in the vectors of bounds $a$ and $b$. All weighted Kolmogorov-Smirnov confidence bands and the nonparametric likelihood confidence bands of Owen (1995) have the form $C B(a, b) \in \mathcal{B}_{V}$ for appropriate choices of $a$ and $b$.

The class $\mathcal{B}_{V}$ does not include all distribution-free confidence bands; it excludes, for example, randomized confidence bands. One may argue, however, that the class includes all distribution-free confidence bands suitable for use in practice. For example, $\mathcal{B}_{V}$ contains all bands whose upper and lower bounds are functions of the EDF $F_{n}(x)$, which is often called the nonparametric sufficient statistic. In addition, Lehmann (1997, pp. 326-328) showed that $\mathcal{B}_{V}$ contains all confidence bands that are equivariant under a continuous, strictly increasing transformation of the data.

Given vectors of bounds $a$ and $b$ that satisfy (1), we may compute the coverage probability of $C B(a, b)$ for continuous $F$ as follows. Consider the interval $\left[X_{(0)}, X_{(1)}\right)$. A necessary and sufficient condition for $F$ not to pass above the upper bound $b_{0}$ on this interval is that $F\left(X_{(1)}\right) \leq b_{0}$. Similarly, a necessary and sufficient condition for $F$ not to pass below the lower bound $a_{0}=0$ on this interval is that $F\left(X_{(0)}\right) \geq$ $a_{0}$. The lower bound condition is thus met trivially on this particular interval, but not in general. A similar pair of conditions is obtained for each of the intervals $\left[X_{(i)}, X_{(i+1)}\right), i=1, \ldots, n$, and we may write that

$$
P_{F}(F \in C B(a, b))=P_{F}\left(a_{1} \leq F\left(X_{(1)}\right) \leq b_{0}, \ldots, a_{n} \leq F\left(X_{(n)}\right) \leq b_{n-1}\right) .
$$

Since $F$ is continuous, the vector $\left(F\left(X_{(1)}\right), \ldots, F\left(X_{(n)}\right)\right)$ is distributed exactly like a 
vector of order statistics from the standard uniform distribution. Thus, we may write the coverage probability in the form

$$
P_{F}(F \in C B(a, b))=P\left(a_{1} \leq U_{(1)} \leq b_{0}, \ldots, a_{n} \leq U_{(n)} \leq b_{n-1}\right),
$$

where $\left(U_{(1)}, \ldots, U_{(n)}\right)$ is a vector of standard uniform order statistics. Probabilities like (2) may be computed in a variety of ways, with perhaps the quickest method being to apply the recursion developed by Noé (1972).

The coverage probabilities that we obtain using (2) are correct provided that $F$ is continuous. When $F$ fails to be continuous, the coverage probabilities obtained from (2) are conservative.

\section{A Class of Optimality Criteria}

In most of the literature on distribution-free confidence bands for a distribution function, the emphasis is on finding confidence bands that have good power when used in the context of goodness-of-fit testing. However, if power is really the goal, then much better tests are available than the ones that can be inverted to give confidence bands

for F. For example, the Neyman-Pearson Lemma (see Lehmann 1997, pp. 74-76) allows one to find the most powerful test of the point null hypothesis $H_{0}: F=F_{0}$ against any simple alternative. One might argue instead that statisticians producing confidence bands for $F$ intend to use the bands as exploratory analysis tools or as tools for simultaneous inference on multiple aspects of $F$ rather than just as tools for goodness-of-fit testing. 
When we think about a confidence band for $F$ as an exploratory analysis tool or a tool for simultaneous inference, it becomes clear that the quality of a given band is a function of how narrow that band is. Since the bands in the class $\mathcal{B}_{V}$ are of fixed width between pairs of consecutive order statistics, the $n+1$ differences $b_{0}-$ $a_{0}, \ldots, b_{n}-a_{n}$ summarize what there is to say about the narrowness of the band. One optimality criterion would be $\sum_{i=0}^{n}\left(b_{i}-a_{i}\right)$, which just totals the differences between the upper and lower bounds of the confidence band on each interval $\left[X_{(i)}, X_{(i+1)}\right)$. More generally, one might choose a vector of positive weights $\left(w_{0}, \ldots, w_{n}\right)$ and seek to minimize $\sum_{i} w_{i}\left(b_{i}-a_{i}\right)$ over all bands $C B(a, b) \in \mathcal{B}_{V}$ with a specified coverage probability. Choosing the weights on the extremes to be large would indicate special interest in having the band be narrow at the extremes. Similarly, choosing the weights to be large in the middle would indicate special interest in having the band be narrow in the middle.

Suppose that $F$ is the distribution function for a distribution known to have bounded support on $[c, d]$. Suppose further that $\left(w_{0}, \ldots, w_{n}\right)$ is chosen by setting $w_{0}=E_{F}\left[X_{(1)}\right]-c, w_{1}=E_{F}\left[X_{(2)}-X_{(1)}\right], \ldots, w_{n}=d-E_{F}\left[X_{(n)}\right]$. A band that minimizes $\sum_{i} w_{i}\left(b_{i}-a_{i}\right)$ then minimizes the expected area enclosed by the band. In particular, a band that minimizes $\sum_{i}\left(b_{i}-a_{i}\right)$ minimizes the expected area when $F$ is the standard uniform distribution function. 


\section{Theoretical Results}

In this section, we consider the problem of minimizing $\sum_{i} w_{i}\left(b_{i}-a_{i}\right)$ over all bands $C B(a, b) \in \mathcal{B}_{V}$ with fixed coverage probability $\alpha \in(0,1)$. We will prove that a minimizer must exist, that any minimizer must satisfy a small set of necessary and sufficient conditions, and that the minimizer will be unique under conditions that are often met in practice. To accomplish this, we first need to prove some results about coverage probabilities.

We defined $V$ to be the set of all vectors $(a, b)=\left(a_{1}, \ldots, a_{n}, b_{0}, \ldots, b_{n-1}\right) \in[0,1]^{2 n}$ that satisfy the conditions (1). Note that $V$ is a compact set. We define the coverage probability function $H: V \rightarrow[0,1]$ by setting

$$
H(a, b) \equiv P_{F}(F \in C B(a, b))
$$

where $F$ is any continuous distribution function. Using (2), we may write $H$ in the integral form

$$
H\left(a_{1}, \ldots, a_{n}, b_{0}, \ldots, b_{n-1}\right)=n ! \int_{u_{1}=a_{1}}^{b_{0}} \ldots \int_{u_{n}=a_{n}}^{b_{n-1}} I\left(u_{1} \leq \ldots \leq u_{n}\right) d u_{n} \ldots d u_{1}
$$

We now consider differentiability properties of $H$. We use the upper-case letters $A_{1}, \ldots, A_{n}, B_{0}, \ldots, B_{n-1}$ to indicate the bounds of a confidence bound as variables and the corresponding lower-case letters to indicate particular values for the bounds. We first consider differentiation of $H$ with respect to a lower bound. Suppose that $a_{i}$ is a lower bound such that $a_{i}<a_{i+1}$ and $a_{i}<b_{i-1}$. Then if $a_{i}$ is increased by a sufficiently small amount $\epsilon>0$, the modified vector of bounds is still in $V$. The 
resulting change in $H$ is given by

$$
\begin{aligned}
\Delta(\epsilon) \equiv & H\left(a_{1}, \ldots, a_{i}+\epsilon, \ldots, b_{n-1}\right)-H\left(a_{1}, \ldots, a_{i}, \ldots, b_{n-1}\right) \\
= & n ! \int_{u_{1}=a_{1}}^{b_{0}} \ldots \int_{u_{i}=a_{i}+\epsilon}^{b_{i}} \ldots \int_{u_{n}=a_{n}}^{b_{n-1}} I\left(u_{1} \leq \ldots \leq u_{n}\right) d u_{n} \ldots d u_{1}- \\
& n ! \int_{u_{1}=a_{1}}^{b_{0}} \ldots \int_{u_{i}=a_{i}}^{b_{i}} \ldots \int_{u_{n}=a_{n}}^{b_{n-1}} I\left(u_{1} \leq \ldots \leq u_{n}\right) d u_{n} \ldots d u_{1} \\
= & -n ! \int_{u_{1}=a_{1}}^{b_{0}} \ldots \int_{u_{i}=a_{i}}^{a_{i}+\epsilon} \ldots \int_{u_{n}=a_{n}}^{b_{n-1}} I\left(u_{1} \leq \ldots \leq u_{n}\right) d u_{n} \ldots d u_{1} .
\end{aligned}
$$

If we then take $\lim _{\epsilon \rightarrow 0} \frac{\Delta(\epsilon)}{\epsilon}$, we find that $\left.\frac{\partial H}{\partial A_{i}^{+}}\right|_{(A, B)=(a, b)}$ is given by

$$
\begin{aligned}
& -n ! \int_{a_{1}}^{b_{0} \wedge a_{i}} \ldots \int_{a_{i-1}}^{b_{i-2} \wedge a_{i}} \int_{a_{i+1}}^{b_{i}} \ldots \int_{a_{n}}^{b_{n-1}} \\
& I\left(u_{1} \leq \ldots u_{i-1} \leq u_{i+1} \ldots \leq u_{n}\right) d u_{n} \ldots d u_{i+1} d u_{i-1} d u_{1},
\end{aligned}
$$

where $x \wedge y=\min \{x, y\}$. Note that this derivative is the sort of quantity that can be computed using Noé's recursion. A similar argument shows that if $a_{i}$ satisfies $a_{i}>a_{i-1}$, then $\left.\frac{\partial H}{\partial A_{i}^{-}}\right|_{(A, B)=(a, b)}$ is also given by (4). Thus, the left-hand and right-hand partial derivatives coincide when both exist.

Applying the same argument to upper bounds $b_{j}$ shows that whenever $b_{j}<b_{j+1}$, $\left.\frac{\partial H}{\partial B_{j}^{+}}\right|_{(A, B)=(a, b)}$ is given by

$$
\begin{aligned}
& n ! \int_{a_{1}}^{b_{0}} \ldots \int_{a_{j}}^{b_{j-1}} \int_{a_{j+2} \vee b_{j}}^{b_{j+1}} \ldots \int_{a_{n} \vee b_{j}}^{b_{n-1}} \\
& I\left(u_{1} \leq \ldots u_{j} \leq u_{j+2} \ldots \leq u_{n}\right) d u_{n} \ldots d u_{j+2} d u_{j} \ldots d u_{1},
\end{aligned}
$$

where $x \vee y=\max \{x, y\}$. Similarly, if $b_{j}$ satisfies $b_{j}>b_{j-1}$ and $b_{j}>a_{j+1}$, then $\left.\frac{\partial H}{\partial B_{j}^{-}}\right|_{(A, B)=(a, b)}$ is given by (5), meaning that the left-hand and right-hand partial derivatives again coincide when both exist. Putting these results together and noting that the arguments $a_{1}, \ldots, a_{n}, b_{0}, \ldots, b_{n-1}$ appear continuously in (4) and (5), we have shown the following theorem. 
Theorem 1. The function $H$ has one-sided partial derivatives with respect to each lower bound $A_{i}$ and each upper bound $B_{j}$ at every point $(a, b) \in V$ where modifying the bound in question by an arbitrarily small amount in the direction of interest does not take the vector of bounds out of $V$. The left-hand and right-hand partial derivatives coincide when they both exist. Moreover, on the domain where a given left-hand or right-hand partial derivative exists, it is continuous in each of the variables $A_{1}, \ldots, A_{n}, B_{0}, \ldots, B_{n-1}$.

Theorem 1 implies that $H$ is continuous in $V$. More importantly, because of the continuity of the one-sided partial derivatives when they exist, Theorem 1 allows us to write the change in coverage probability resulting from multiple infinitesimal changes in the bounds in terms of the partial derivatives of $H$ at the starting point.

Theorem 2. Let $(a, b) \in V$, and let $\delta=\left(\delta_{a_{1}}, \ldots, \delta_{b_{n-1}}\right) \in \mathbb{R}^{2 n}$ be a fixed vector. Suppose that there exists $r>0$ small enough that whenever $0<\epsilon<r$, adding $\epsilon \delta$ to $(a, b)$ results in another point in $V$. Then, if $\left.\frac{\partial H}{\partial A_{i}}\right|_{(A, B)=(a, b)}$ and $\left.\frac{\partial H}{\partial B_{j}}\right|_{(A, B)=(a, b)}$ are the partial derivatives of $H$ with respect to the bounds $A_{i}$ and $B_{j}$ in the directions corresponding to $\delta_{a_{i}}$ and $\delta_{b_{j}}$, we have that

$$
\begin{gathered}
H\left(a_{1}+\epsilon \delta_{a_{1}}, \ldots, b_{n-1}+\epsilon \delta_{b_{n-1}}\right) \\
=H\left(a_{1}, \ldots, b_{n-1}\right)+\epsilon\left(\left.\delta_{a_{1}} \frac{\partial H}{\partial A_{1}}\right|_{(A, B)=(a, b)}+\ldots+\left.\delta_{b_{n-1}} \frac{\partial H}{\partial B_{n-1}}\right|_{(A, B)=(a, b)}\right)+o(\epsilon) \cdot(6)
\end{gathered}
$$

The following theorem uses the continuity of $H$ to show that an optimal confidence band must exist.

Theorem 3. Let $\left(w_{0}, \ldots, w_{n}\right)$ be a vector of positive weights, and let $\alpha \in(0,1)$ be 
a specified coverage probability. Then there exists a confidence band that minimizes $\sum_{i} w_{i}\left(b_{i}-a_{i}\right)$ over the set of all confidence bands $C B(a, b) \in \mathcal{B}_{V}$ such that $H(a, b)=\alpha$.

Proof of Theorem 3. Since $H$ is continuous, and since both coverage probability 0 and coverage probability 1 are attainable in $V$, the set $\{(a, b) \in V: H(a, b)=\alpha\}$ is nonempty. It is also compact. Since the function $(a, b) \rightarrow \sum_{i} w_{i}\left(b_{i}-a_{i}\right)$ is continuous, it must achieve a minimum on this compact set.

We now develop necessary and sufficient conditions for a given confidence band to be optimal. The next theorem shows that if $C B(a, b) \in \mathcal{B}_{V}$ is optimal at level $\alpha$, then the lower bounds $a_{1}, \ldots, a_{n}$ and the upper bounds $b_{0}, \ldots, b_{n-1}$ are strictly increasing as opposed to merely nondecreasing. To show this, we first need a lemma that will also be used later. This lemma shows that for a fixed vector $\left(w_{0}, \ldots, w_{n}\right)$, the optimal value of $\sum_{i} w_{i}\left(b_{i}-a_{i}\right)$ is a strictly increasing function of the level $\alpha$.

Lemma 1. If $C B(a, b) \in \mathcal{B}_{V}$ is optimal with respect to the weights $\left(w_{0}, \ldots, w_{n}\right)$, then no confidence band in $\mathcal{B}_{V}$ can have the same value as $C B(a, b)$ for the optimality criterion and yet have a higher coverage probability.

Proof of Lemma 1. We proceed by proving the contrapositive. Let $C B(a, b) \in \mathcal{B}_{V}$ have coverage probability $\alpha \in(0,1)$, and suppose that $C B(c, d) \in \mathcal{B}_{V}$ is such that $\sum_{i} w_{i}\left(b_{i}-a_{i}\right)=\sum_{i} w_{i}\left(d_{i}-c_{i}\right)$, but $H(c, d)>H(a, b)=\alpha$. Since $H(c, d)>0$, we must have $d_{0}>c_{1}$. If we lower $d_{0}$ towards $c_{1}$, then the modified vector of bounds remains in $V$. As we decrease $d_{0}$, we decrease $\sum_{i} w_{i}\left(d_{i}-c_{i}\right)$, and we also decrease the coverage probability from $H(c, d)>\alpha$ at the start to 0 once $d_{0}=c_{1}$. Since $H$ is a continuous, there must exist $d^{\star} \in\left(c_{1}, d_{0}\right)$ such that $H\left(c_{1}, \ldots, c_{n}, d^{\star}, d_{1}, \ldots, d_{n-1}\right)=\alpha$. Since this 
new band has the same coverage probability $\alpha$ as $C B(a, b)$, but a smaller value for the optimality criterion, $C B(a, b)$ is not optimal with respect to the criterion with weights $\left(w_{0}, \ldots, w_{n}\right)$.

Theorem 4. Suppose that $C B(a, b) \in \mathcal{B}_{V}$ is optimal at level $\alpha \in(0,1)$ with respect to the criterion with weights $\left(w_{0}, \ldots, w_{n}\right)$. Then $a_{1}<\ldots<a_{n}$, and $b_{0}<\ldots<b_{n-1}$.

Proof of Theorem 4. Suppose that there exists $i \in\{1,2, \ldots, n-1\}$ such that $a_{i}=$ $a_{i+1}$. Then there exists either a positive integer $l$ such that $a_{i}=\ldots=a_{i+l}<a_{i+l+1}<$ 1 or a positive integer $l$ such that $a_{i}=\ldots=a_{i+l}<1$ and $i+l=n$. In either case, the last inequality (involving 1) follows from the fact that $\alpha=H(a, b)>0$. By (4), $\left.\frac{\partial H}{\partial A_{i+l}^{+}}\right|_{(A, B)=(a, b)}=0$, while by $(4),\left.\frac{\partial H}{\partial B_{0}^{-}}\right|_{(A, B)=(a, b)}>0$. If we increase $a_{i+l}$ by $\epsilon / w_{i+l}$ and decrease $b_{0}$ by $\epsilon / w_{0}$ for small enough $\epsilon>0$ that the modified vector of bounds remains in $V$, then the optimality criterion is unchanged. However, by the expansion (6), the coverage probability changes by $\left.\epsilon \frac{\partial H}{\partial B_{0}^{-}}\right|_{(A, B)=(a, b)} / w_{0}+o(\epsilon)$, which is positive for sufficiently small $\epsilon$. Thus, by Lemma 1 , the confidence band $C B(a, b)$ cannot be optimal. The proof that $b_{0}, \ldots, b_{n-1}$ are strictly ordered is completely analogous.

The next theorem gives necessary conditions for $C B(a, b) \in \mathcal{B}_{V}$ to be optimal.

Theorem 5. Suppose that $C B(a, b) \in \mathcal{B}_{V}$ is optimal at level $\alpha$ with respect to the criterion with weights $\left(w_{0}, \ldots, w_{n}\right)$. Then there exists a constant $C>0$ such that for each lower bound $a_{i}>0$,

$$
\left.\frac{\partial H}{\partial A_{i}}\right|_{(A, B)=(a, b)}=-C w_{i}
$$


and for each upper bound $b_{j}<1$,

$$
\left.\frac{\partial H}{\partial B_{j}}\right|_{(A, B)=(a, b)}=C w_{j}
$$

If $a_{1}=0$, then

$$
\left.\frac{\partial H}{\partial A_{1}^{+}}\right|_{(A, B)=(a, b)} \leq-C w_{1}
$$

and if $b_{n-1}=1$, then

$$
\left.\frac{\partial H}{\partial B_{n-1}^{-}}\right|_{(A, B)=(a, b)} \geq C w_{n-1} .
$$

Proof of Theorem 5. This proof uses the same type of argument used to prove Theorem 4 . Let $a_{i}$ be a lower bound, $i=2, \ldots, n$. Then, since $C B(a, b)$ is optimal, the lower bounds are strictly ordered, and we can change the value of $a_{i}$ slightly in either direction without leaving $V$. We can also change the value of $b_{0}$ slightly in either direction without leaving $V$. If we decrease $a_{i}$ by $\epsilon / w_{i}$ and decrease $b_{0}$ by $\epsilon / w_{0}$ for small $\epsilon$ (positive or negative), then the optimality criterion is unchanged, but by the expansion (6), the coverage probability changes by

$$
\epsilon\left(-\left.\frac{\partial H}{\partial A_{i}}\right|_{(A, B)=(a, b)} / w_{i}-\left.\frac{\partial H}{\partial B_{0}}\right|_{(A, B)=(a, b)} / w_{0}\right)+o(\epsilon) .
$$

Since $C B(a, b)$ is optimal, (7) must always be nonpositive. But since $\epsilon$ can be positive or negative, (7) can only always be nonpositive if the quantity in parentheses, which is free of $\epsilon$, is equal to 0 . This proves that

$$
\left.\frac{\partial H}{\partial A_{i}}\right|_{(A, B)=(a, b)}=-\left(\left.\frac{\partial H}{\partial B_{0}}\right|_{(A, B)=(a, b)} / w_{0}\right) w_{i} \equiv-C w_{i} .
$$

The case of $b_{j}<1$ follows by a similar argument.

Suppose that $a_{1}=0$. Then, since the lower bounds $a_{1}, \ldots, a_{n}$ are strictly ordered, we can increase the value of $a_{1}$ slightly without leaving $V$. We can also increase the 
value of $b_{0}$ slightly without leaving $V$. If we increase $a_{1}$ by $\epsilon / w_{1}>0$ and increase $b_{0}$ by $\epsilon / w_{0}$, then the optimality criterion is unchanged, but the coverage probability changes by

$$
\epsilon\left(\left.\frac{\partial H}{\partial A_{1}^{+}}\right|_{(A, B)=(a, b)} / w_{1}+\left.\frac{\partial H}{\partial B_{0}}\right|_{(A, B)=(a, b)} / w_{0}\right)+o(\epsilon)
$$

Since $C B(a, b)$ is optimal, (8) must always be nonpositive. But since we can choose $\epsilon>0$ to be arbitrarily small, (8) can only always be nonpositive if the quantity in parentheses, which is free of $\epsilon$, is nonpositive. This proves that

$$
\left.\frac{\partial H}{\partial A_{1}^{+}}\right|_{(A, B)=(a, b)} \leq-\left(\left.\frac{\partial H}{\partial B_{0}}\right|_{(A, B)=(a, b)} / w_{0}\right) w_{1}=-C w_{1} .
$$

The case of $b_{n-1}=1$ follows similarly.

It is natural to ask whether the necessary conditions derived in Theorem 5 are also sufficient for $C B(a, b)$ to be optimal. It turns out that they are. To show this, we use an important result, the Brunn-Minkowski Inequality, from the theory of convex bodies. Proofs of this inequality are given in Leichtweiss (1998) and many other sources in the mathematics literature. Gardner (2002) gives a very readable study of the Brunn-Minkowski Inequality, its history, and its relationship to other well-known inequalities in analysis and geometry, while Das Gupta (1980) describes applications of the inequality in statistics. We note that the convex combination $(1-\lambda) K_{1}+\lambda K_{2}$ of the sets $K_{1}$ and $K_{2}$ is defined by

$$
(1-\lambda) K_{1}+\lambda K_{2} \equiv\left\{(1-\lambda) x+\lambda y: x \in K_{1}, y \in K_{2}\right\}
$$


Theorem 6 (Brunn-Minkowski). Let $K_{1}$ and $K_{2}$ be bounded convex sets in $\mathbb{R}^{n}$, and let $\lambda \in(0,1)$. Then if $V_{n}(\cdot)$ is the volume function in $\mathbb{R}^{n}$, we have that

$$
V_{n}\left((1-\lambda) K_{1}+\lambda K_{2}\right) \geq\left((1-\lambda) V_{n}\left(K_{1}\right)^{1 / n}+\lambda V_{n}\left(K_{2}\right)^{1 / n}\right)^{n} .
$$

If both $V_{n}\left(K_{1}\right)$ and $V_{n}\left(K_{2}\right)$ are positive, then equality holds in (9) iff

$$
K_{2}=\rho K_{1}+t
$$

for $\rho>0$ and $t \in \mathbb{R}^{n}$.

Theorem 6 holds under the weaker hypothesis that $K_{1}$ and $K_{2}$ are merely measurable rather than convex, provided that $\lambda K_{1}+(1-\lambda) K_{2}$ is also measurable (see, for example, Gardner 2002). For this paper, however, we need only the classical version of the inequality. The Brunn-Minkowski Inequality is relevant to our situation because any $C B(a, b)$ can be associated with a convex set $K(a, b) \subset \mathbb{R}^{n}$. Specifically, $C B(a, b)$ may be associated with the set

$$
K(a, b) \equiv\left\{\left(x_{1}, \ldots, x_{n}\right): a_{1} \leq x_{1} \leq b_{0}, \ldots, a_{n} \leq x_{n} \leq b_{n-1}, 0 \leq x_{1} \leq \cdots \leq x_{n} \leq 1\right\} .
$$

This set $K(a, b)$ is bounded, and it is convex because it is the intersection of a finite collection of half-spaces. By (2), the coverage probability of $C B(a, b)$ is just $n ! V_{n}(K(a, b))$. We note that each set $K(a, b)$ is the intersection of an $n$-dimensional rectangle with the $n$-dimensional simplex

$$
\Omega=\left\{\left(x_{1}, \ldots, x_{n}\right): 0 \leq x_{1} \leq \cdots \leq x_{n} \leq 1\right\}
$$

We also note that if $(a, b),(c, d) \in V$ and $\lambda \in(0,1)$, then

$$
(1-\lambda) K(a, b)+\lambda K(c, d) \subset K((1-\lambda) a+\lambda c,(1-\lambda) b+\lambda d) .
$$


Using the association between $C B(a, b)$ and $K(a, b)$, we can show that the necessary conditions for optimality given in Theorem 5 are also sufficient.

Theorem 7. Suppose that $C B(a, b) \in \mathcal{B}_{V}$ satisfies the conditions given in Theorem 5. Then $C B(a, b)$ is optimal at level $\alpha$ with respect to the criterion with weights $\left(w_{0}, \ldots, w_{n}\right)$

Proof of Theorem 7. We prove the result by contradiction. Suppose that there exists a confidence band with the same coverage probability as $C B(a, b)$, but a strictly smaller value for the optimality criterion. Then, by Lemma 1, there must exist $C B(c, d) \in \mathcal{B}_{V}$ such that $\sum_{i} w_{i}\left(b_{i}-a_{i}\right)=\sum_{i} w_{i}\left(d_{i}-c_{i}\right)$, but $H(c, d)>H(a, b)$. Let $\lambda \in(0,1)$. Then, by (12), the convex combination $(1-\lambda) K(a, b)+\lambda K(c, d)$ is contained in the convex set associated with the band $C B((1-\lambda) a+\lambda c,(1-\lambda) b+\lambda d)$. For this band, the value of the optimality criterion is

$$
\begin{aligned}
& \sum_{i} w_{i}\left((1-\lambda) b_{i}+\lambda d_{i}-\left((1-\lambda) a_{i}+\lambda c_{i}\right)\right) \\
= & (1-\lambda) \sum_{i} w_{i}\left(b_{i}-a_{i}\right)+\lambda \sum_{i} w_{i}\left(d_{i}-c_{i}\right) \\
= & \sum_{i} w_{i}\left(b_{i}-a_{i}\right),
\end{aligned}
$$

which is the same, for any $\lambda$, as the value of the optimality criterion for $C B(a, b)$ and $C B(c, d)$. Define a function

$$
G(\lambda) \equiv H((1-\lambda) a+\lambda c,(1-\lambda) b+\lambda d) \geq n ! V_{n}((1-\lambda) K(a, b)+\lambda K(c, d)) .
$$

This function is differentiable via the chain rule since $H$ is differentiable, and $G(0)=$ $n ! V_{n}(K(a, b))$. Thus, we have by the Brunn-Minkowski Inequality that

$$
\left.\frac{d G}{d \lambda}\right|_{\lambda=0} \geq\left. n ! \frac{d}{d \lambda} V_{n}((1-\lambda) K(a, b)+\lambda K(c, d))\right|_{\lambda=0}
$$




$$
\begin{aligned}
& \geq\left.\frac{d}{d \lambda}\left((1-\lambda) H(a, b)^{1 / n}+\lambda H(c, d)^{1 / n}\right)^{n}\right|_{\lambda=0} \\
& \geq\left. n\left((1-\lambda) H(a, b)^{1 / n}+\lambda H(c, d)^{1 / n}\right)^{n-1}\left(H(c, d)^{1 / n}-H(a, b)^{1 / n}\right)\right|_{\lambda=0} \\
& =\left(n\left(H(a, b)^{1 / n}\right)^{n-1}\left(H(c, d)^{1 / n}-H(a, b)^{1 / n}\right)\right)>0 .
\end{aligned}
$$

This derivative $\left.\frac{d G}{d \lambda}\right|_{\lambda=0}$ can also be computed by noting that for sufficiently small $\lambda$, we can use (6) to write $\left.\frac{d G}{d \lambda}\right|_{\lambda=0}$ in terms of the partial derivatives of $H$ at $(a, b)$. Specifically, letting the vector $\delta$ of small changes in the bounds be given by

$$
\delta=\left(c_{1}-a_{1}, \ldots, c_{n}-a_{n}, d_{0}-b_{0}, \ldots, d_{n-1}-b_{n-1}\right)
$$

we have that if neither a bound $a_{1}=0$ nor a bound $b_{n-1}=1$ is changed, then changing $(a, b)$ by $\epsilon \delta$ for $\epsilon>0$ changes the coverage probability by

$\epsilon\left(-\left(c_{1}-a_{1}\right) C w_{1}-\ldots-\left(c_{n}-a_{n}\right) C w_{n}+\left(d_{0}-b_{0}\right) C w_{0}+\ldots+\left(d_{n-1}-b_{n-1}\right) C w_{n-1}\right)+o(\epsilon)$,

which is just

$$
\epsilon C\left(\sum_{i=0}^{n} w_{i}\left(d_{i}-c_{i}\right)-\sum_{i=0}^{n} w_{i}\left(b_{i}-a_{i}\right)\right)+o(\epsilon)=o(\epsilon)
$$

But then $\left.\frac{d G}{d \lambda}\right|_{\lambda=0}=0$, whereas the Brunn-Minkowski Inequality told us that $\left.\frac{d G}{d \lambda}\right|_{\lambda=0}>$ 0 . If a bound $a_{1}=0$ or $b_{n-1}=1$ is changed, then $\left.\frac{d G}{d \lambda}\right|_{\lambda=0} \leq 0$. In either case, we have a contradiction. Thus, $C B(a, b)$ is optimal at level $\alpha$ with respect to the criterion with weights $\left(w_{0}, \ldots, w_{n}\right)$.

Now that necessary and sufficient conditions for optimality have been determined, we may wonder whether optimal bands are unique. The Brunn-Minkowski Inequality is again the key to obtaining useful results. 
Theorem 8. Suppose that $C B(a, b), C B(c, d) \in \mathcal{B}_{V}$ are each optimal at level $\alpha$ with respect to the criterion with weights $\left(w_{0}, \ldots, w_{n}\right)$. Then $K(a, b)$ and $K(c, d)$ differ only by a translation.

Proof of Theorem 8. Note first that if $K(a, b)$ and $K(c, d)$ are different in any way, then taking any convex combination of them yields a new convex set $(1-\lambda) K(a, b)+$ $\lambda K(c, d) \subset K((1-\lambda) a+\lambda c,(1-\lambda) b+\lambda d)$, where $C B((1-\lambda) a+\lambda c,(1-\lambda) b+\lambda d)$ has the same value for the optimality criterion as $C B(a, b)$ and $C B(c, d)$. By the BrunnMinkowski Inequality, this new band must have a coverage probability strictly larger than $\alpha$ unless the equality condition (10) holds. Since both $C B(a, b)$ and $C B(c, d)$ are optimal, the new band cannot have a higher coverage probability. Thus, $K(a, b)$ and $K(c, d)$ are related as $K(c, d)=\rho K(a, b)+t$ for some $\rho>0$ and $t \in \mathbb{R}^{n}$. Since $C B(c, d)$ and $C B(a, b)$ have the same coverage probability, $K(a, b)$ and $K(c, d)$ have the same volume. Hence $\rho=1$. Thus, $K(a, b)$ and $K(c, d)$ differ only by a translation.

The next result further clarifies the conditions under which more than one optimal band may exist.

Theorem 9. Suppose that $C B(a, b) \in \mathcal{B}_{V}$ is optimal at level $\alpha$ with respect to the criterion with weights $\left(w_{0}, \ldots, w_{n}\right)$. If $\left(a_{i}, b_{i-1}\right)$ overlaps with $\left(a_{i+1}, b_{i}\right)$ for $i=$ $1, \ldots, n-1$, or in other words if

$$
a_{i+1}<b_{i-1}, i=1, \ldots, n-1,
$$

then $C B(a, b)$ is the unique optimal band up to translation of every bound by the same real constant. 
Proof of Theorem 9. Recall that $K(a, b)$ is the intersection of an $n$-dimensional rectangle and the $n$-dimensional simplex $\Omega$ defined in (11). An arbitrary translation of such a set will not lead to another set of the same type. Suppose that we translate $\left(x_{1}, \ldots, x_{n}\right)$ by $t=\left(t_{1}, \ldots, t_{n}\right)$, obtaining a new vector of variables $y_{i}=$ $x_{i}+t_{i}, i=1, \ldots, n$. Then, if $\left(x_{1}, \ldots, x_{n}\right)$ satisfies $a_{i} \leq x_{i} \leq b_{i-1}, i=1, \ldots, n$, the vector $\left(y_{1}, \ldots, y_{n}\right)$ satisfies $a_{i}+t_{i} \leq y_{i} \leq b_{i-1}+t_{i}, i=1, \ldots, n$. However, even if $\left(x_{1}, \ldots, x_{n}\right)$ satisfies $x_{i} \leq x_{i+1}, i=1, \ldots, n-1$, the vector $\left(y_{1}, \ldots, y_{n}\right)$ only satisfies $y_{i}-t_{i} \leq y_{i+1}-t_{i+1}, i=1, \ldots, n-1$. In other words, $\left(y_{1}, \ldots, y_{n}\right)$ satisfies $y_{i} \leq y_{i+1}+\left(t_{i}-t_{i+1}\right), i=1, \ldots, n-1$. The constraints that $\left(y_{1}, \ldots, y_{n}\right)$ satisfies do not define a convex set associated with a confidence band unless for each $i=1, \ldots, n-1$, either $t_{i}=t_{i+1}$ or the condition $x_{i} \leq x_{i+1}$ is redundant in that it is implied by the constraints $a_{i} \leq x_{i} \leq b_{i-1}$ and $a_{i+1} \leq x_{i+1} \leq b_{i}$ on $x_{i}$ and $x_{i+1}$. If the interval $\left(a_{i}, b_{i-1}\right)$ overlaps with the interval $\left(a_{i+1}, b_{i}\right)$ for each $i=1, \ldots, n-1$, then none of the conditions $x_{i} \leq x_{i+1}, i=1, \ldots, n-1$ are redundant, meaning that $t_{1}=\ldots=t_{n}$ must hold.

This theorem leads directly to a number of results indicating which kinds of optimal confidence bands are unique.

Corollary 1. Suppose that $C B(a, b) \in \mathcal{B}_{V}$ is optimal at level $\alpha$ with respect to the criterion with weights $\left(w_{0}, \ldots, w_{n}\right)$. If $w_{0} \neq w_{n}$ and condition (13) is satisfied, then $C B(a, b)$ is the unique optimal band.

Proof of Corollary 1. Since (13) holds, every other optimal band arises from adding a constant value $s \neq 0$ to each of the bounds for $C B(a, b)$. But if $s$ is added to each 
of the bounds, then the value of the optimality criterion becomes

$$
\begin{aligned}
& w_{0}\left(b_{0}+s\right)+\sum_{i=1}^{n-1} w_{i}\left(b_{i}+s-\left(a_{i}+s\right)\right)+w_{n}\left(1-\left(a_{n}+s\right)\right) \\
= & \sum_{i} w_{i}\left(b_{i}-a_{i}\right)+s\left(w_{0}-w_{n}\right)
\end{aligned}
$$

since the values $a_{0}=0$ and $b_{n}=1$ are fixed. This value (14) coincides with $\sum_{i} w_{i}\left(b_{i}-\right.$ $a_{i}$ ) only if $w_{0}=w_{n}$.

Corollary 2. Suppose that $C B(a, b) \in \mathcal{B}_{V}$ is optimal at level $\alpha$ with respect to the criterion with weights $\left(w_{0}, \ldots, w_{n}\right)$, and suppose that condition (13) is satisfied. If $a_{1}=0$ and $b_{n-1}=1$, then $C B(a, b)$ is the unique optimal band.

Proof of Corollary 2. If $s>0$ is added to $b_{n-1}=1$ or $s<0$ is added to $a_{1}=0$, the resulting bound is not located in the interval $[0,1]$.

Corollary 3. Suppose that $C B(a, b) \in \mathcal{B}_{V}$ is optimal at level $\alpha$ with respect to the criterion with weights $\left(w_{0}, \ldots, w_{n}\right)$, and suppose that condition (13) is satisfied. If $C B(a, b)$ is symmetric in the sense that $a_{i}+b_{n-i}=1, i=1, \ldots, n$, then $C B(a, b)$ is the unique symmetric optimal band.

Proof of Corollary 3. It suffices to note that translating all bounds by the same constant $s \neq 0$ would destroy the symmetry of the band $C B(a, b)$.

The final result in this section tells us that if we use a vector of weights $\left(w_{0}, \ldots, w_{n}\right)$ that is symmetric in the sense that $w_{i}=w_{n-i}$ for $i=0, \ldots, n$, then there must exist a symmetric optimal confidence band at any desired level.

Theorem 10. Suppose that $\left(w_{0}, \ldots, w_{n}\right)$ satisfies $w_{i}=w_{n-i}$ for $i=0, \ldots, n$, and suppose that $\alpha \in(0,1)$ is the desired coverage probability. Then there must exist 
$C B(a, b) \in \mathbb{B}_{V}$ that is both symmetric in the sense that $a_{i}+b_{n-i}=1, i=1, \ldots, n$, and optimal at level $\alpha$ with respect to the criterion with weights $\left(w_{0}, \ldots, w_{n}\right)$.

Proof of Theorem 10. By Theorem 3, there must exist an optimal band $C B(c, d) \in$ $\mathcal{B}_{V}$. Consider the band $C B(e, f)$ with lower bounds given by $\left(e_{1}, \ldots, e_{n}\right)=(1-$ $\left.d_{n-1}, \ldots, 1-d_{0}\right)$ and upper bounds given by $\left(f_{0}, \ldots, f_{n-1}\right)=\left(1-c_{n}, \ldots, 1-c_{1}\right)$. Since a vector of standard uniform order statistics $\left(U_{(1)}, \ldots, U_{(n)}\right)$ satisfies

$$
\left(U_{(1)}, \ldots, U_{(n)}\right) \stackrel{d}{=}\left(1-U_{(n)}, \ldots, 1-U_{(1)}\right)
$$

we have that $H(c, d)=H(e, f)$. Moreover, because of the symmetry in $\left(w_{0}, \ldots, w_{n}\right)$, the optimality criterion for the new band is

$$
\sum_{i} w_{i}\left(1-c_{n-i}-\left(1-d_{n-i}\right)\right)=\sum_{i} w_{n-i}\left(d_{n-i}-c_{n-i}\right)=\sum_{i} w_{i}\left(d_{i}-c_{i}\right)
$$

which is the same as for $C B(c, d)$. Thus, $C B(e, f)$ is also optimal. Now consider the band $C B\left(\frac{1}{2}(c+e), \frac{1}{2}(d+f)\right)$. This new band is symmetric by construction, and the Brunn-Minkowski Inequality shows that it is optimal.

\section{A Computational Algorithm}

Through the theoretical results obtained in Section 4, we know that optimal confidence bands exist, that they are unique under certain conditions, and that they can be recognized as optimal based on the partial derivatives of the coverage probability function $H$. What is still lacking, however, is a computational algorithm for finding optimal bands when given $\alpha$ and $\left(w_{0}, \ldots, w_{n}\right)$. This section provides such an algorithm. The motivation for this algorithm is the observation that one can move 
towards satisfying the conditions specified in Theorem 5 if one relaxes bounds that lead to high values of the quotients $\left|\frac{\partial H}{\partial A_{i}} / w_{i}\right|$ or $\left|\frac{\partial H}{\partial B_{j}} / w_{j}\right|$ and tightens bounds that lead to low values of those quotients. Note that in this particular optimization problem, it is impossible to have a locally optimal band that is not also globally optimal. This removes one of the usual concerns in an optimization problem.

\section{Computational Algorithm:}

Step 1: Choose a starting band. One possibility would be to use the nonparametric likelihood confidence band with the desired coverage probability $\alpha$.

Step 2: Specify a small starting step size, say $h=(4 n)^{-1}$, which gives the size of the moves that will be made initially.

Step 3: Compute $\frac{d H}{d A_{1}}, \ldots, \frac{d H}{d A_{n}}, \frac{d H}{d B_{0}}, \ldots, \frac{d H}{d B_{n-1}}$ at the current confidence band. Update these partial derivatives whenever the band is changed. Computational time can be saved by noticing that changing one bound does not noticeably change derivatives other than those corresponding to bounds near the bound that was changed.

Step 4: When the current coverage probability is less than or equal to $\alpha$, increase the coverage probability by increasing (if it is an upper bound) or decreasing (if it is a lower bound) by $h$ the moveable bound with the highest value of $\left|\frac{\partial H}{\partial A_{i}} / w_{i}\right|$ or $\left|\frac{\partial H}{\partial B_{j}} / w_{j}\right|$. A bound is moveable if making a change of size $h$ in the specified direction does not take the vector of bounds out of $V$.

Step 5: When the current coverage probability is greater than $\alpha$, decrease the coverage probability by decreasing (if it is an upper bound) or increasing (if it is a lower bound) by $h$ the moveable bound with the lowest value of $\left|\frac{\partial H}{\partial A_{i}} / w_{i}\right|$ or $\left|\frac{\partial H}{\partial B_{j}} / w_{j}\right|$. 
Step 6: If the most recent move has returned the band to a band already visited at the current step size $h$, decrease $h$ by a factor of two to avoid an infinite loop.

Step 7: Repeat Steps 4 to 6, keeping Step 3 in mind, until a prespecified stopping criterion is met. The criterion that we use looks at the size of $h$.

For the case of symmetric weights $\left(w_{0}, \ldots, w_{n}\right)$, Theorem 10 guarantees that a symmetric optimal confidence band will exist. It then suffices to work with only the lower bounds, taking the upper bounds to be given by $b_{i}=1-a_{n-i}, i=0, \ldots, n$.

\section{Shape and Power Comparisons}

Though narrowness of the confidence band was the motivation for the optimality criteria discussed in Section 3, it is of interest to compare the performance of the optimal bands to that of other confidence bands both in terms of narrowness and in terms of power. The bands chosen for comparison with the optimal bands were (a) the Kolmogorov-Smirnov band, (b) the Anderson-Darling band based on the weighted Kolmogorov-Smirnov statistic

$$
D_{A D}=\sup _{x} \frac{\left|F_{n}(x)-F_{0}(x)\right|}{\sqrt{F_{0}(x)\left(1-F_{0}(x)\right)}},
$$

and (c) the nonparametric likelihood confidence bands proposed by Owen (1995). Optimal bands based on three different choices of weights were used in the comparison. The first choice of weights was uniform weights $(1, \ldots, 1)$. The second choice of weights, made to emphasize narrowness of the band in the extremes, set $w_{i}=100$ 
for the outermost $n / 5$ intervals on either extreme and $w_{i}=1$ for the rest. The final choice, made to de-emphasize narrowness of the band in the extremes, set $w_{i}=1$ for the outermost $n / 5$ intervals on either extreme and $w_{i}=100$ for the rest.

Symmetric bands with coverage probability $95 \%$ were produced for sample sizes $n=20$ and $n=40$. These bands are plotted for the case of equispaced data in Figures 1 and 2. Note that since (13) is met for each of the bands shown, the optimal bands are unique. A number of important features of both the optimal bands and their competitors are evident from these plots. We see that the Kolmogorov-Smirnov band has a uniform width except near the extremes, that the Anderson-Darling band is so wide that one almost suspects that the coverage probability has been computed incorrectly, and that the nonparametric likelihood band has a roughly elliptical shape. The plots for the three optimal bands show that different choices of weights can lead to substantially different optimal bands. The band enclosing the smallest area, of course, is the band that is optimal with respect to the criterion with uniform weights.

\section{Include Figures 1 and 2 here.}

To compare power properties of the bands, a class of alternative distributions that includes both heavy-tailed and light-tailed distributions was used. The standard uniform distribution was used as the null hypothesis distribution, and the alternatives considered were the $\operatorname{Beta}(\eta, \eta)$ distributions for $\log (\eta) \in(-2,2)$. When $\eta$ is small, the distribution $\operatorname{Beta}(\eta, \eta)$ has heavy tails, while when $\eta$ is large, the tails are light. The case $\eta=1$, of course, gives the standard uniform distribution. Plots of power versus $\log (\eta)$ for the bands plotted in Figures 1 and 2 are given in Figures 3 and 4. The 
nonparametric likelihood band seems to have the best overall power performance, with the performance of the optimal band with weights emphasizing the extremes being comparable. The Anderson-Darling band has the best power against heavytailed alternatives, but it accomplishes this at the cost of having almost no power against light-tailed alternatives. The optimal confidence bands are comparable to the best of their competitors in terms of power.

\section{Include Figures 3 and 4 here.}

As another illustration of the optimal bands, we applied them and the competitor bands to a collection of galaxy velocities given in Table 1 of Roeder (1990). This data set, which includes 82 values, was also considered by Owen (1995). The resulting bands are given in Figure 5, where the units for velocity are thousands of kilometers per second.

\section{$\overline{\text { Include Figure } 5 \text { here. }}$}

\section{Conclusions}

We have shown how to obtain distribution-free confidence bands for a distribution function that are optimal with respect to any of a class of narrowness criteria. Unlike other distribution-free confidence bands in the literature, these bands do not arise from test inversion, though of course each band is associated with a hypothesis test. Instead, these bands are motivated by exploratory analysis considerations that suggest that narrowness, rather than power, is the important criterion. That power and 
narrowness may be conflicting goals is best seen by considering the Anderson-Darling bands. They have good power against heavy-tailed alternatives (see Figures 3 and 4), but they are extremely wide (see Figures 1, 2, and 5). One nice property of the optimal bands is that by making appropriate choices of the weights $\left(w_{0}, \ldots, w_{n}\right)$, one may obtain bands that are narrow in whatever region of the distribution is of interest.

\section{References}

[1] Anderson, T. W. and Darling, D. A. (1952). Asymptotic theory of certain "goodness of fit" criteria based on stochastic processes, Annals of Mathematical Statistics, 23, 193-212.

[2] Berk, R. H. and Jones, D. H. (1979). Goodness-of-fit test statistics that dominate the Kolmogorov statistics, Zeitschrift für Wahrenscheinlichkeitstheorie und Verwandte Gebiete, 47, 47-59.

[3] Das Gupta, S. (1980). Brunn-Minkowski inequality and its aftermath, Journal of Multivariate Analysis, 10, 296-318.

[4] Gardner, R. J. (2002). The Brunn-Minkowski inequality, Bulletin of the American Mathematical Society, 39, 355-405.

[5] Leichtweiss, K. (1998). Affine Geometry of Convex Bodies, Heidelberg: Barth.

[6] Lehmann, E. L. (1997). Testing Statistical Hypotheses, 2nd ed., New York: Springer-Verlag. 
[7] Noé, M. (1972). The calculation of distributions of two-sided KolmogorovSmirnov type statistics, Annals of Mathematical Statistics, 43, 58-64.

[8] Owen, A. (1995). Nonparametric likelihood confidence bands for a distribution function, Journal of the American Statistical Association, 90, 516-521.

[9] Roeder, K. (1990). Density estimation with confidence sets exemplified by superclusters and voids in the galaxies, Journal of the American Statistical Association, 85, 617-624. 
(a)

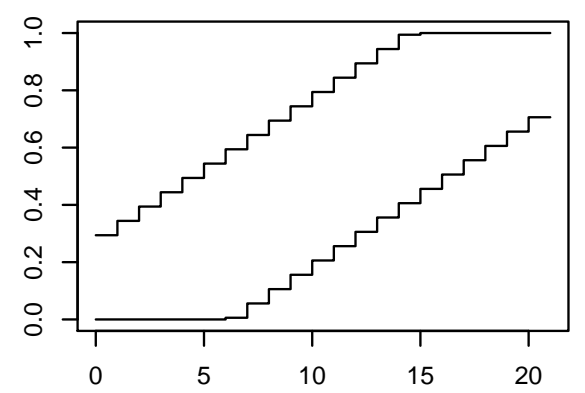

(c)

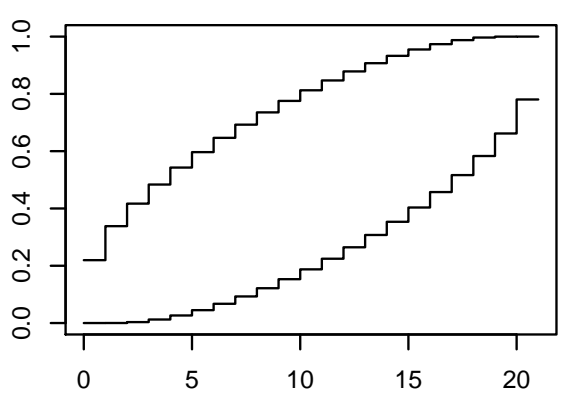

(e)

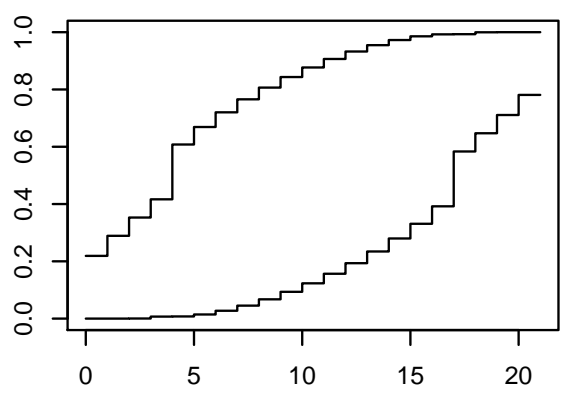

(b)

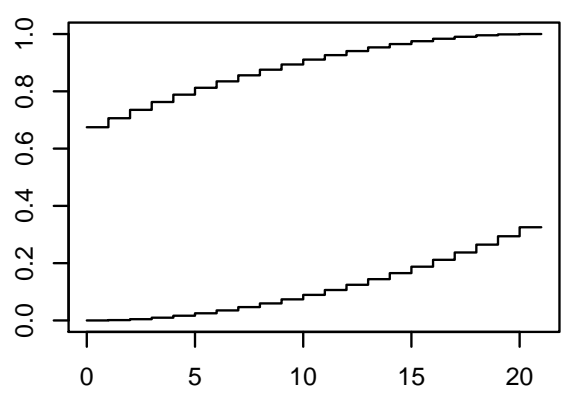

(d)

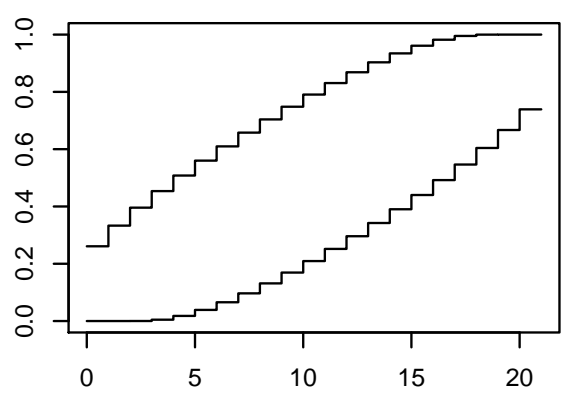

(f)

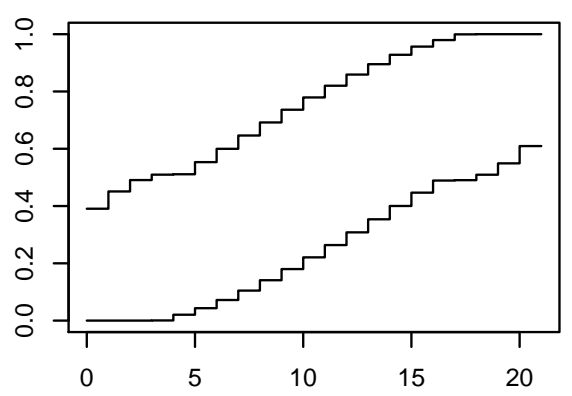

Figure 1: Six different 95\% confidence bands when $n=20$. The bands are (a) Kolmogorov-Smirnov, (b) Anderson-Darling, (c) nonparametric likelihood, (d) uniform-weight optimal, (e) optimal with weights emphasizing the extremes, and (f) optimal with weights de-emphasizing the extremes. 
(a)

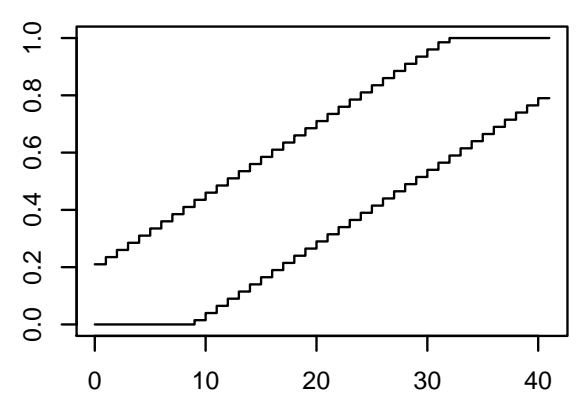

(c)

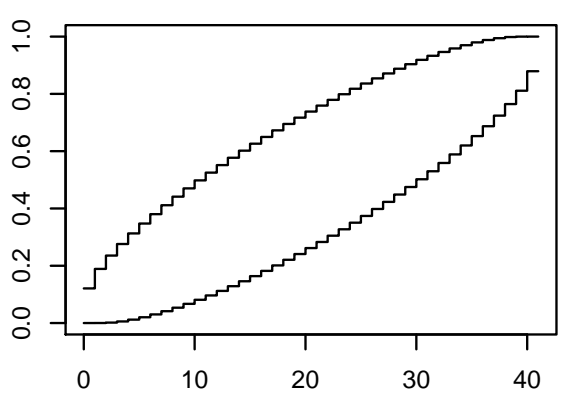

(e)

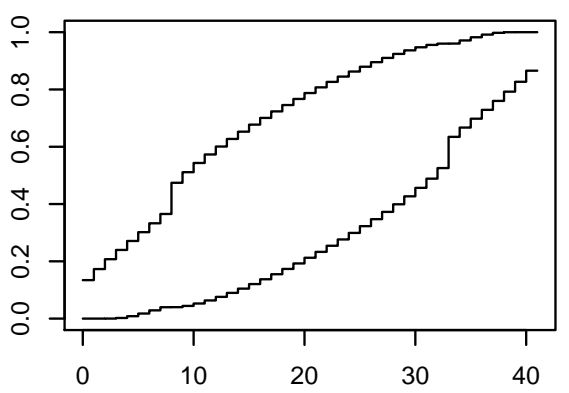

(b)

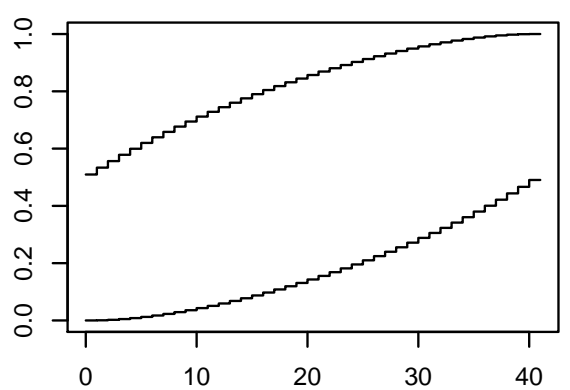

(d)

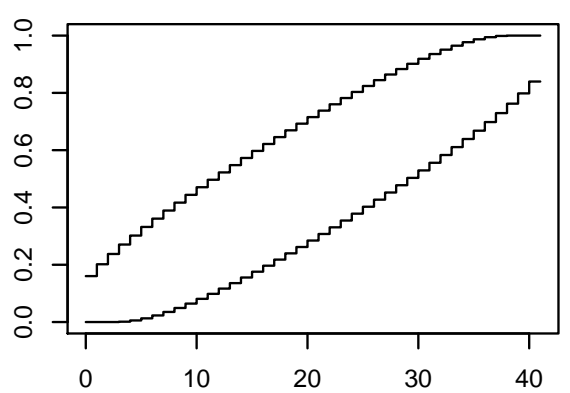

(f)

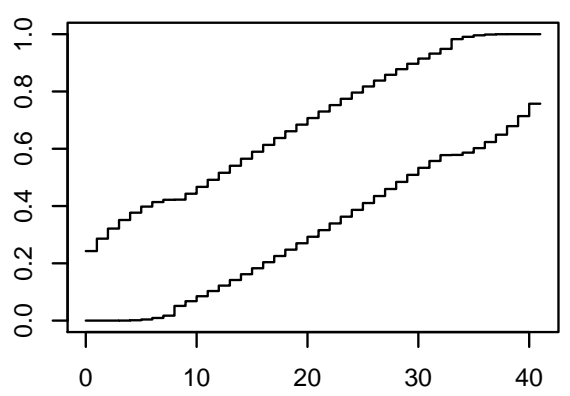

Figure 2: Six different $95 \%$ confidence bands when $n=40$. The bands are (a) Kolmogorov-Smirnov, (b) Anderson-Darling, (c) nonparametric likelihood, (d) uniform-weight optimal, (e) optimal with weights emphasizing the extremes, and (f) optimal with weights de-emphasizing the extremes. 
(a)

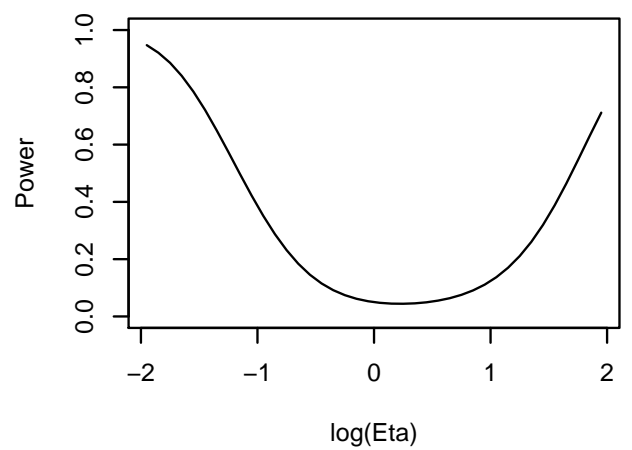

(c)

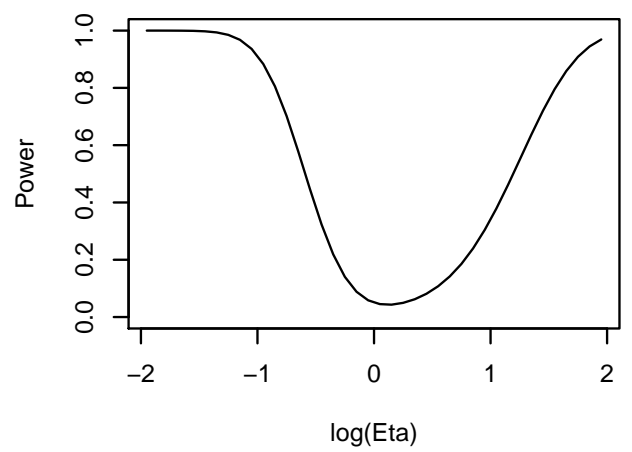

(e)

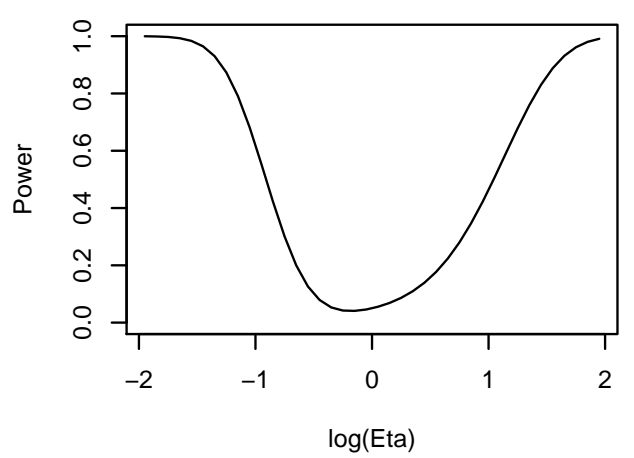

(b)

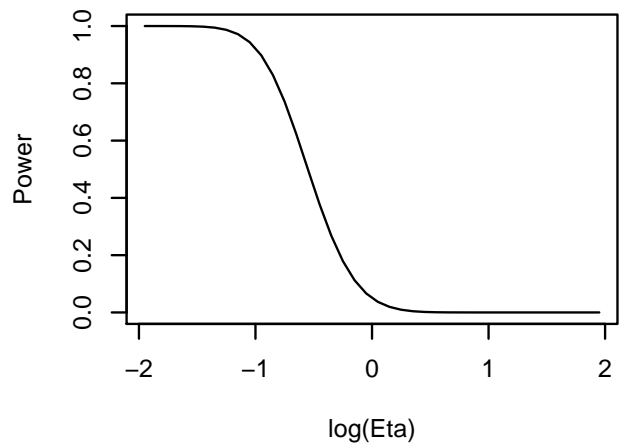

(d)

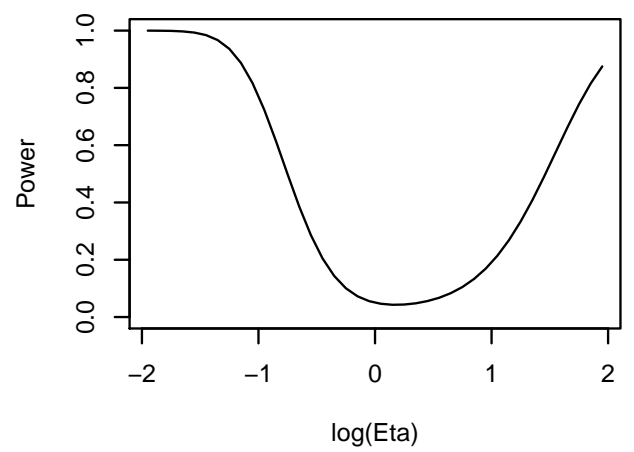

(f)

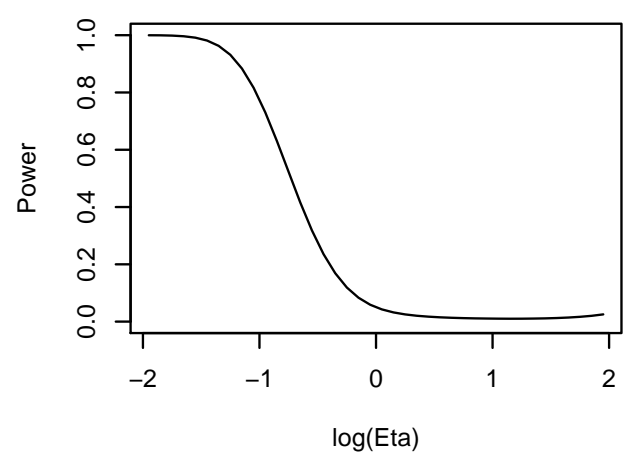

Figure 3: Power curves for the $95 \%$ confidence bands when $n=20$. The alternative is the $\operatorname{Bet} a(\eta, \eta)$ distribution. The bands are (a) Kolmogorov-Smirnov, (b) AndersonDarling, (c) nonparametric likelihood, (d) uniform-weight optimal, (e) optimal with weights emphasizing the extremes, and (f) optimal with weights de-emphasizing the extremes. 
(a)

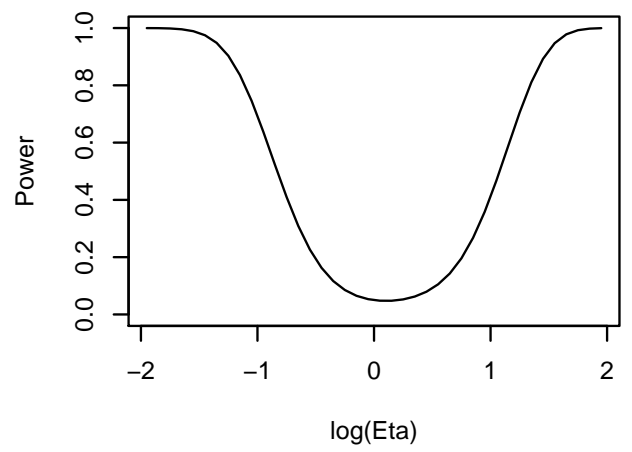

(c)

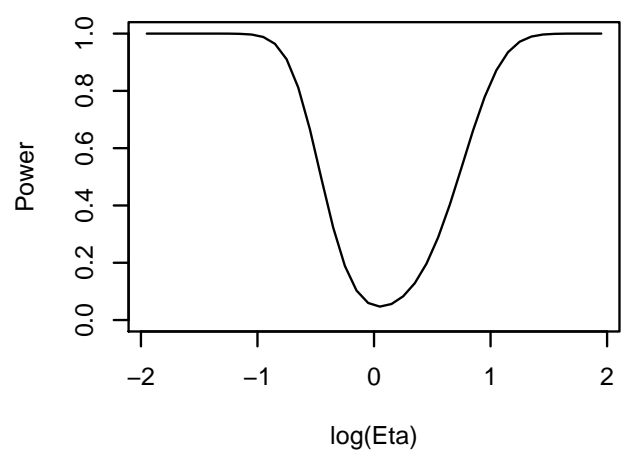

(e)

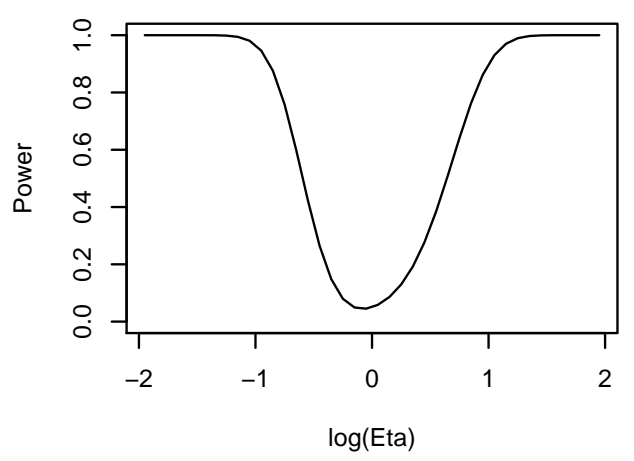

(b)

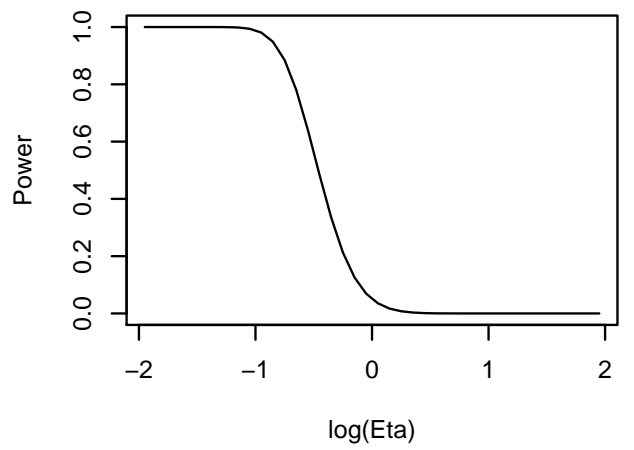

(d)

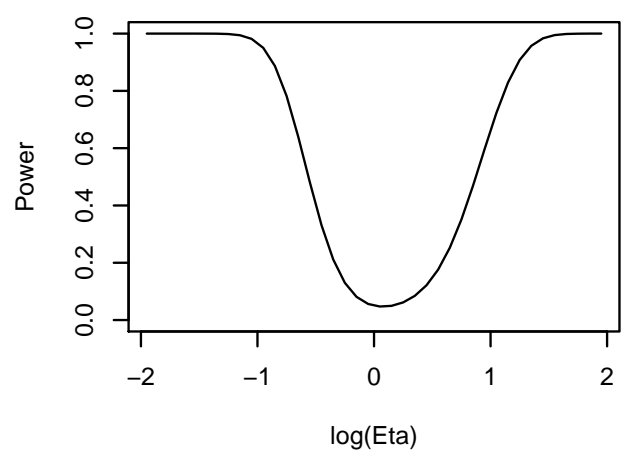

(f)

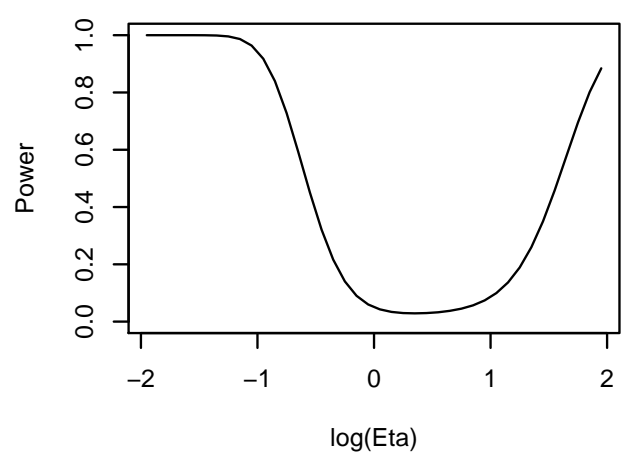

Figure 4: Power curves for the $95 \%$ confidence bands when $n=40$. The alternative is the $\operatorname{Bet} a(\eta, \eta)$ distribution. The bands are (a) Kolmogorov-Smirnov, (b) AndersonDarling, (c) nonparametric likelihood, (d) uniform-weight optimal, (e) optimal with weights emphasizing the extremes, and (f) optimal with weights de-emphasizing the extremes. 
(a)

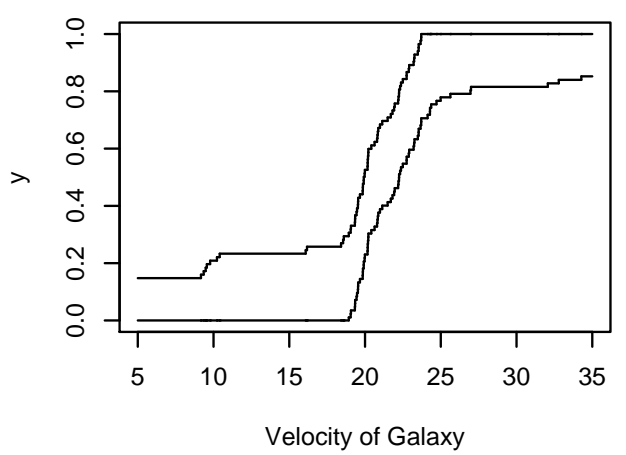

(c)

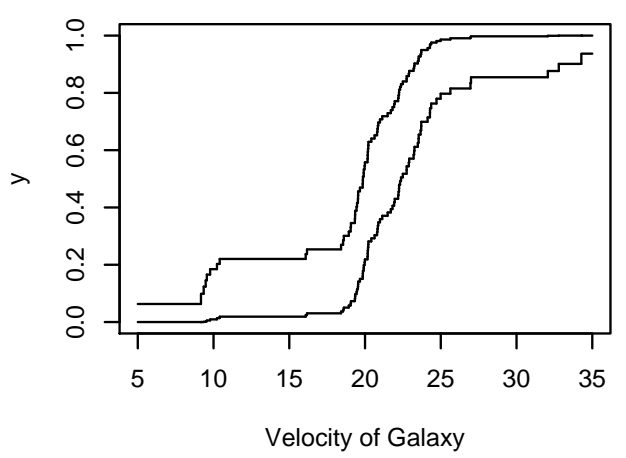

(e)

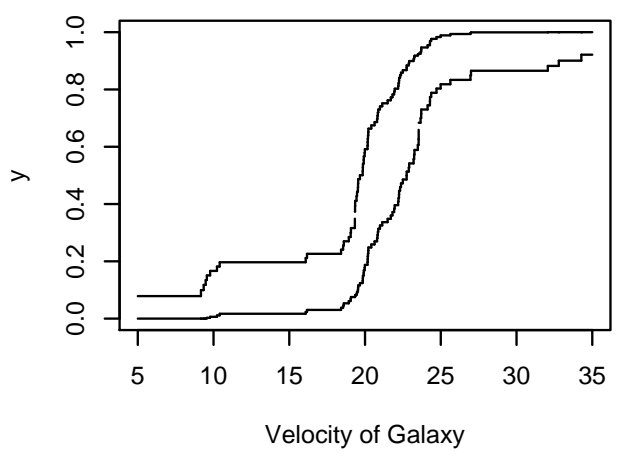

(b)

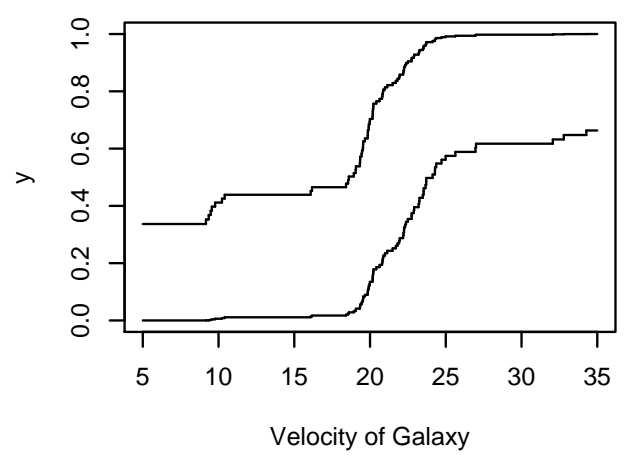

(d)

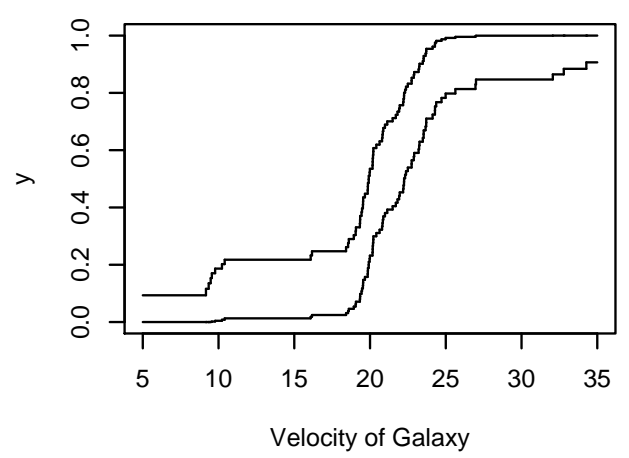

(f)

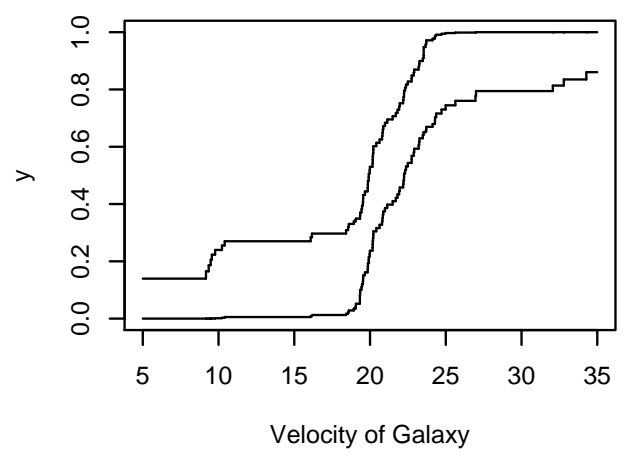

Figure 5: Six different 95\% confidence bands for the galaxy velocity data. The bands are (a) Kolmogorov-Smirnov, (b) Anderson-Darling, (c) nonparametric likelihood, (d) uniform-weight optimal, (e) optimal with weights emphasizing the extremes, and (f) optimal with weights de-emphasizing the extremes. 